\title{
Multi-Band CAP for Next Generation Optical Access Networks Using 10-G Optics
}

\author{
(Invited Paper)
}

\author{
Jinlong Wei, Senior Member, IEEE, and Elias Giacoumidis, Member, IEEE
}

\begin{abstract}
Gb/s/ $\lambda$ multi-band carrierless amplitude and phase (CAP) modulation long reach passive optical networks (LR-PONs) were demonstrated using 10-G class transceivers only. A major issue of multi-band CAP is that it is vulnerable to timing error and this work has offered quantitative analysis about it in detail for the first time. A novel simple timing recovery approach, partial differential QAM constellation encoding and decoding schemes together with blind multi-modulus algorithms (MMAs) equalization are proposed to address the issue efficiently, enabling zero-overhead signal recovery. Results show that it can offer excellent system tolerance to timing error of at least $\mathbf{\pm 0 . 1}$ symbol period even for the highest frequency CAP sub-band. The characteristics of the transceiver are measured and optimization of critical system parameters is performed including the CAP sub-band count, 10-G Mach-Zehnder intensity modulator operation conditions, optical launch power, and wavelength offset asymmetrical optical filtering. For downlink using erbium-doped fiber amplifier (EDFA) pre-amplifiers, successful $40-\mathrm{Gb} / \mathrm{s}$ multi-band CAP signal transmission over an 80-km (90-km) SMF is achieved with a link power budget of $33 \mathrm{~dB}(29 \mathrm{~dB})$ considering a FEC threshold BER of $3.8 \times 10^{-3}$.
\end{abstract}

Index Terms-Long reach passive optical network, Carrierless amplitude and phase modulation, Modulation format, Multi-modulus algorithm, Partial differential QAM, Optical power budget, Blind equalization, Timing error, Timing recovery, phase ambiguity, cycle slip.

\section{INTRODUCTION}

A lthough the industry is ready to deploy the fastest standardized $40-\mathrm{Gb} / \mathrm{s}$ time and wavelength division multiplexing passive optical networks (TWDM-PONs) [1], the next generation (NG)-PONs beyond $40-\mathrm{Gb} / \mathrm{s}$ have been intensively investigated featuring $25 \mathrm{~Gb} / \mathrm{s}$ or $40 \mathrm{~Gb} / \mathrm{s}$ lane rate or beyond as a result of the continuous rise in broadband service demand. For example, the IEEE 802.3 Ethernet Working Group is discussing on 4 channels with $25 \mathrm{~Gb} / \mathrm{s}$ lane rate as an option

Manuscript received ?, 2017; Revised 2017; Accepted ?, 2017.

Jinlong Wei is now with Huawei Technologies Duesseldorf $\mathrm{GmbH}$, European Research Center, Riesstrasse 25, 80992 München, Germany (jinlong.wei@huawei.com). He was with ADVA Optical Networking SE, Märzenquelle 1-3, 98617 Meiningen, Germany.

Elias Giacoumidis is now with both the School of Electronic Engineering, Dublin City University, The Rince Institute, Dublin 9, Irland and the SFI research center CONNECT, 34 Westland Row, Trinity College Dublin, Dublin 2, Ireland (eliasgiacoumidis@hotmail.com).

Copyright (c) 2015 IEEE. for NG-EPON [2]. This is mainly fueled by bandwidth-hungry applications such as high definition TV and video-on-demand as well as emerging services like virtual reality and cloud computing. In addition, the 5G Infrastructure Public Private Partnership (5G PPP) anticipates that 5G mobile networks need to accommodate a 1000-fold increase in data traffic thus low-cost mobile front-haul traffic will become one of the major drivers for PON data rates exceeding $10 \mathrm{~Gb} / \mathrm{s}[3,4]$. Focus is on low-cost solutions using advanced single carrier modulation schemes and demonstrations have been performed including chirp managed NRZ [5-7], electrical/optical Duobinary [8-10], and PAM-4 $[8,11]$. These schemes have shown the feasibility of supporting $25 \mathrm{~Gb} / \mathrm{s}$ or $40 \mathrm{~Gb} / \mathrm{s}$ data rate over typically $20-\mathrm{km}$ SMF using 10-G transmitter [7-11] and/or 10-G receiver [7-9], thus are capable of offering low-cost solutions.

In addition to use of low cost optics, high speed long reach PONs (LR-PONs) are key technologies to facilitate metro/access and fixed-mobile convergences to further reduce cost and power [12]. This is critcal for cost and power saving from operators' point of view. At high bit rates of $40 \mathrm{~Gb} / \mathrm{s} / \lambda$, however, the above-mentioned single carrier schemes with simple linear or nonlinear equalizations $[10,11,13,14]$ are difficult to support transmission beyond 20-km SMF with reasonably high optical link power budgets unless dedicated dispersion compensation fibers (DCFs) [8,9] are adopted. The use of DCFs increases system cost and insertion loss, reduces system flexibility and complicates link configuration. On the contrary, multi-carrier $[15,16]$ and/or multi-band schemes $[17,18]$ featuring enhanced spectral efficiency and flexibility by fully leveraging advanced digital signal process (DSP) can support high capacity and long reach without altering the optical infrastructure. In addition, simple asymmetrical optical filtering by utilizing the deployed wavelength division multiplexing (WDM) multiplexers (MUXs) and de-multiplexers (De-MUXs) or Arrayed waveguide gratings (AWGs) is advantageous for multi-band or multi-carrier systems to increase fiber dispersion tolerance [16-18]. As indicated in [7], such optical filtering approach can achieve stable performance in field trial demonstrations.

High speed single- or multi-band carrierless amplitude and phase (CAP) modulation systems have been intensively investigated for a potential low cost solutions for medium and long reach PONs [18-21]. Nevertheless, none of these demonstrations except [19] paid much attention to the 


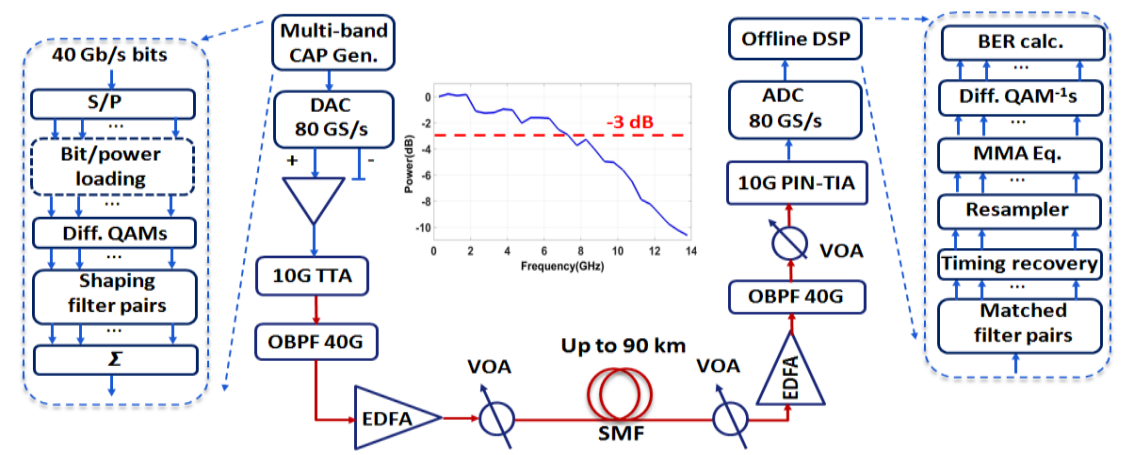

Fig. 1. Experimental setup of a $40 \mathrm{~Gb} / \mathrm{s}$ lane rate NG-PON downstream link using multi-band CAP. The inset shows the 3-dB bandwidth measurement of the system for an optical back to back link.

significant technical issue of the strong sensitivity of CAP signals to timing jitter. This is an intrinsic disadvantage for CAP especially when multiple bands are implemented [22]. Although training symbols-assisted phase tracking and estimation in the receiver side can recover the signal [18,21], it introduces overhead and additionally high frequency non-data dependent jitter beyond the phase tacking loop bandwidth cannot be tracked or compensated. We have proposed partial differential quadrature amplitude modulation (QAM) encoding and decoding schemes together with multi-modulus algorithm (MMA) equalization to efficiently recover multi-band CAP signals in a completely blind manner [17, 19]. Based on zero-overhead signal recovery DSPs, an cost-effective optical amplified $40 \mathrm{~Gb} / \mathrm{s}$ lane rate multi-band CAP LR-PON system was experimentally demonstrated using $10-\mathrm{G}$ class transceivers only and it can offer excellent link power budgets [19].

Based on our previous work in [19], this paper significantly extends the experimental demonstration of the $40 \mathrm{~Gb} / \mathrm{s}$ multi-band LR-PON using 10-G optics by looking into not only the system performance, but also the system timing recovery as well as system timing error tolerance. Principles and the encoding and decoding process of high order partial differential QAM schemes are also presented. The characteristics of the optical transceivers are measured and optimization of a number of critical transceiver and link parameters is performed, which includes the 10-G MZM modulator's operating conditions, optical fiber launch power, vestigial sideband (VSB) optical filtering, as well as CAP sub-band count. Results show that 40 $\mathrm{Gb} / \mathrm{s}$ VSB multi-band CAP signal is successfully transmitted over up to $80 \mathrm{~km}(90 \mathrm{~km}) \mathrm{SMF}$ with an optical downlink power budget of $33 \mathrm{~dB}(29 \mathrm{~dB})$ by taking into account a forward error correction (FEC) threshold of $3.8 \times 10^{-3}$.

This work is organized as follows. Section II describes the experimenal setup and looks into the influence of timing jitter to multi-band CAP signals and the principles of partial differential QAM schemes. Section III presents the experimental results including optimization of critical parameters, the system optical power sensivities, and optical link power budgets. Finally, Section IV summarize this work.

\section{EXPERIMENTAL SETUP}

\section{A. Experiment System}

Fig. 1 depicts the setup for a LR $40 \mathrm{~Gb} / \mathrm{s}$ lane rate multi-band CAP NG-PON system experiment. The transmitter contains an offline multi-band CAP signal generator, an 80-GS/s digital-to-analog convertor (DAC), a single-ended linear driver, and a $10-\mathrm{G}$ tunable transmitter assembly (TTA). The TTA consists of a tunable laser (with 50-GHz WDM grid granularity) operating at $1543.3 \mathrm{~nm}$ and an InP dual drive Mach-Zehnder intensity modulator (MZM) with a bandwidth of around 13 $\mathrm{GHz}$ [11]. Although the TTA is more expensive than a 10-G directly modulated laser (DML) [5-7], it keeps a lower system operation and maintanence cost especially when DWDM is considered in green field deployments. Following the MZM, a tunable optical bandpass filter (OBPF) with a 3-dB bandwidth of approximately $39 \mathrm{GHz}$ is adopted and its output is amplified by a booster erbium-doped fiber amplifier (EDFA). The OBPF has a filtering profile very similar to a $50-\mathrm{GHz}$ grid WDM MUX [23]. The OBPF is tuned to generate a frequency offset between the laser frequency and the MUX center frequency, leading to a VSB multi-band CAP signal [17-19]. The VSB signal can significantly increase the tolerance to fiber $\mathrm{CD}$ compared to the double sideband (DSB) case. The frequency offset is optimized in order to maximize the system performance. A variable optical attenuator (VOA) at the output of the booster EDFA adjusts the optical power launched into the fiber link. After transmission over an SMF link up to $90 \mathrm{~km}$, a combined optical receiver consists of VOAs, pre-amplifier EDFA, a 40-GHz bandwidth OBPF with identical configuration of the transmitter OBPF, and a 10-G PIN-TIA is utilized to detect the received optical signal. A VOA is used to optimize the input power injected into the PIN-TIA. Note that the pre-amplifier EDFA can be co-located with and prior to a De-MUX or AWG in the remote node in optical amplified LR-PONs, thus the cost can be shared by multiple subscribers. The detected multi-band CAP signal is then converted into a digital signal by an ADC sampling at $80 \mathrm{GS} / \mathrm{s}$. The digital signal is then sent to a computer and undergoes offline signal processing.

The detailed process of offline DSPs for the transmitter and the receiver is also presented in Fig. 1, which is similar to that presented in [17]. The transmitter offline DSP includes a serial-to-parallel (S/P) convertor, a Levin Campello algorithm-based bit/power loading module needed at the beginning of the communication, differential QAM encoders varying from differential binary phase shift keying (DBPSK), DQPSK to partial differential QAM-32 to convert bit streams into complex symbols, orthogonal square-root raised cosine 


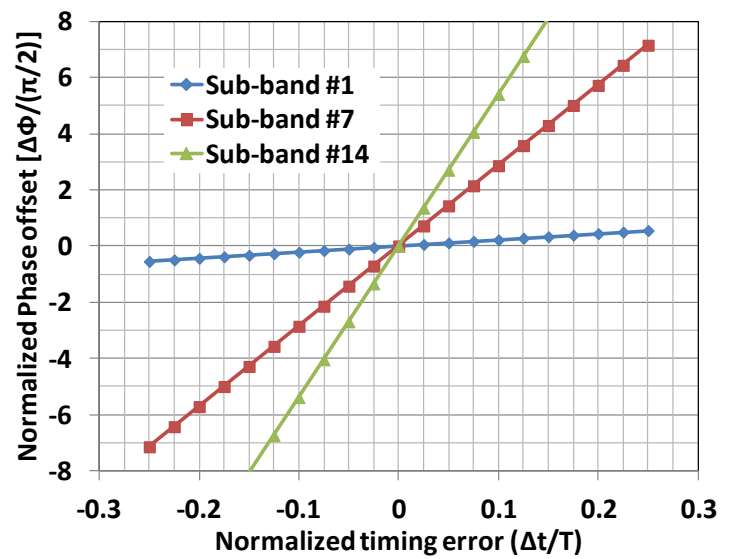

Fig. 2. Normalized phase offset (to $\pi / 2$ ) versus normalized timing error (to symbol time period) for different sub-band signals.

(SRRC) shaping filter pairs containing inphase(I) and quadrature (Q) channels with a roll-off coefficient of $\alpha=0.1$ at its assigned center frequency $f_{k}(k=1,2, \ldots, N)$, and an addition operation to add symbols of all sub-bands. $N$ is the sub-band count. The added signal is then clipped to achieve a peak-to-average ratio of $11 \mathrm{~dB}$ and quantized to the amplitude range (0 to 255) required by the DAC. The receiver offline DSP consists of matched SRRC filter pairs to separate the sub-band signals, a timing recovery module to obtain the optimum sampling point for each sub-band signal, a re-sampler that generates two samples per symbol, a simple blind 14-tap T/2 space ( $T$ is the symbol time period) MMA-based feedforward equalizer (FFE), whose output is directly fed to the following partial differential QAM decoders to convert complex symbols into bit streams. The bit error ratio (BER) prior to FEC is the result of a one-on-one comparison between the transmitted and the recovered bits. The CAP signal baud rate is $1 \mathrm{GBaud}$, leading to an oversampling of 80 . The matched SRRC filters have 880 taps (11 symbols length) and a roll-off coefficient of 0.1. Note that in real implementations, however, the ADC sampling speed can be reduced significantly and the matched filters of sub-bands at lower frequencies need much less number of taps [24].

Note that currently, NG PON bit rates are not yet clear, especially for the 40-G case. We thus assume two possible FEC schemes: a low complex $\operatorname{RS}(255,223)$ with $14.35 \%$ overhead, $6.56 \mathrm{~dB}$ net coding gain and FEC threshold BER of $10^{-3}$, which was adopted by $10 \mathrm{G}-\mathrm{EPON}$ standard, and a more complex high gain hard-decision FEC with threshold BER of $3.8 \times 10^{-3}$ and $7 \%$ - overhead [25]. We denote these two schemes as FEC1 and FEC2, respectively, in this paper. No extra DSP overhead is needed since the receiver DSP is performed in a completely blind manner, as explained in the following subsection. In addition, the wavelength plan for $40-\mathrm{G}$ per wavelength $\mathrm{NG}$ PON is not clear neither. Semiconductor optical amplifier instead of EDFA shown in Fig. 1 could be used if O-band wavelength plan is considered.

\section{B. Timing Recovery}

One of the key contributions of this work is to address the timing recovery issue with multi-band CAP. As indicated in (a) Sub-band \#1

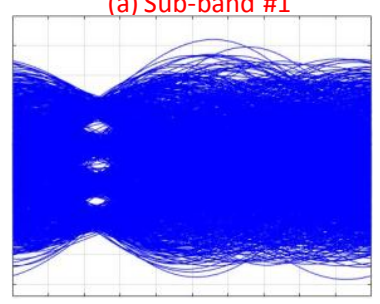

(b) Sub-band \#7

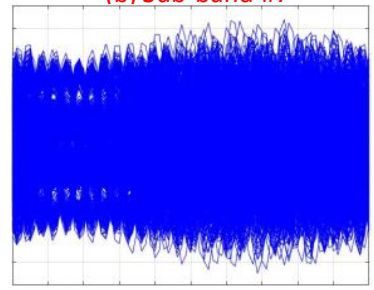

(c) Sub-band \#14
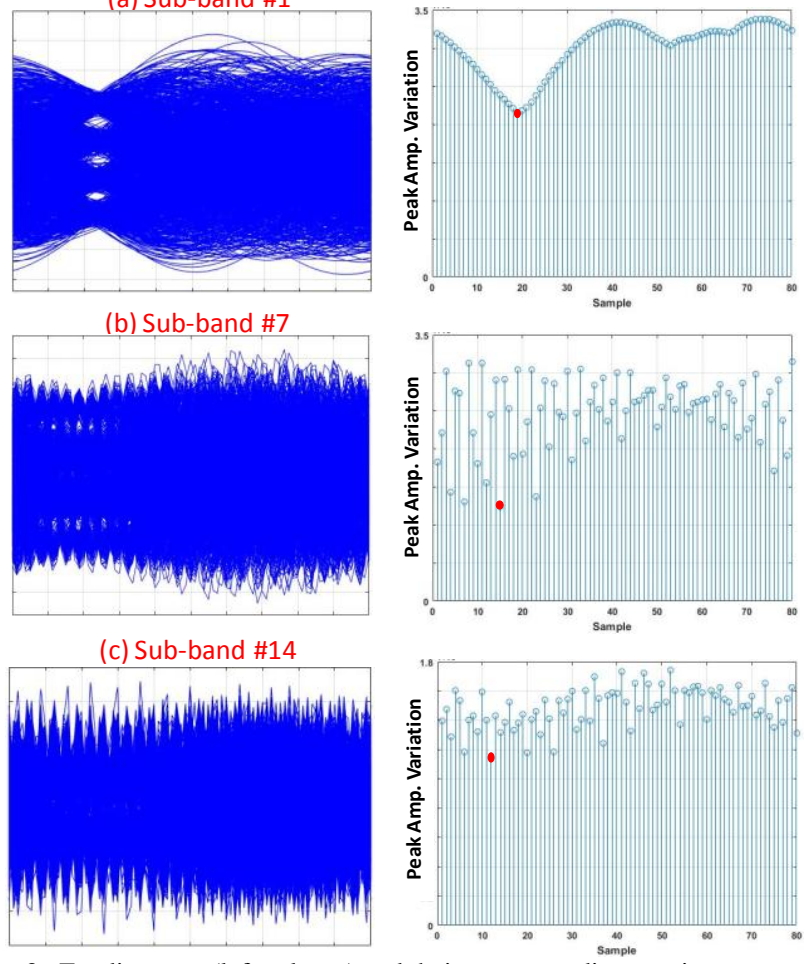

Fig. 3. Eyediagrams (left column) and their corresponding maximum sample amplitude variations (right column) for the $1^{\text {st }}, 7^{\text {th }}$, and $14^{\text {th }}$ sub-band signals after matched filters, respectively. Note that the red solid dot in the right column most likely indicates the center of the eye.

previous work [22], CAP system is sensitive to timing jitter. For multi-band CAP, this technical issue especially applies to sub-bands at high frequencies. The reason can be explained by simple mathematics: a timing error of $\Delta t(-T / 2<\Delta t<T / 2)$ will cause a phase offset of $\Delta \phi_{k}(k=1,2,3, \ldots, N)$ to the $k$-th sub-band received signal constellation after matched filters. The phase offset is given by [22]

$$
\Delta \phi_{k}=2 \pi f_{k} \Delta t
$$

Assuming no guard band between each adjacent sub-bands, the center frequency of the $k$-th sub-band is $f_{k}=(1+\alpha) \cdot(2 k-1) / 2 T$.

Then Eq. (1) can be rewritten as

$$
\Delta \phi_{k}=\pi(1+\alpha)(2 k-1) \frac{\Delta t}{T}
$$

According to Eq. (2), the phase offset is linearly proportional to two factors: 1) the timing error normalized to symbol time period, and 2) the sub-band index. Fig. 2 shows the phase offset dependence on the timing error for various sub-bands. It is very interesting to notice that for sub-bands at high frequencies, a very small timing error can cause significant phase offset. For example, for the $7^{\text {th }}$ and $14^{\text {th }}$ sub-band, timing error of 0.05 symbol period and 0.025 symbol period would bring about over $\pi / 2$ phase offset.

For square QAM constellations, MMA equalization [26,27] is an efficient blind method for constellation recovery which is more cost effective and does not require retraining after sudden channel changes, since the optical channel is not highly time varying. Square QAM constellations are rotationally symmetric 


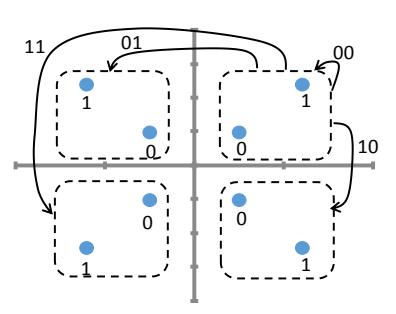

(a) QAM-8

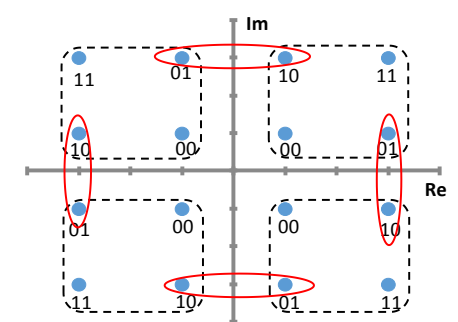

(b) QAM-16

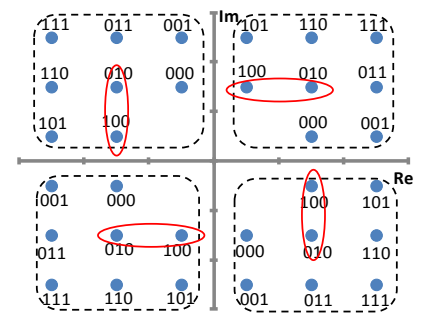

(c) QAM-32

Fig. 4. Partial differential encoding for a square constellation of (a) QAM-8, (b) QAM-16, and (c) QAM-32.

by the angle $\pi / 2$ ( $\pi$ for DBPSK). This means errors that are multiples of $\pi / 2$ in the phase are undetectable. This is so called $\pi / 2$ phase ambiguity that absolute phase rotation of $M \times \pi / 2$ ( $M= \pm 1, \pm 2, \pm 3, \ldots$ and $M \neq 4 j$ and $j$ is an integer number) of the constellation introduced by the channel cannot be recovered by the equalizer, also referred to as cycle slip in the remainder of the paper. This consequently brings about very stringent requirements on timing recovery especially for high frequency sub-bands, otherwise phase offset tracking and estimation must be conducted since timing error is usually time dependent [22].

Fig. 3 left column illustrates the matched filters' output signals whose information will be used to conduct timing recovery via extracting the optimum sample point without aid of training symbols. Since there are no clock tones available in multi-CAP spectrum due to strong Nyquist filtering, the simple conventional square timing recovery approach [28] cannot be adopted here. We propose a simple approach for the optimum timing phase search as shown in Fig. 3 right column. This approach utilizes the statistical property that the optimum sampling point has approximately the lowest peak amplitude variation (PAV) by observing the sample amplitude over a number of symbol periods, as indicated by the eye diagrams in Fig. 3. For $M$ symbol periods, suppose the $i$-th sampling position of each symbol period corresponding to the $k$-th sub-band contains samples $S_{i, k}$, where the range of $i$ is determined by the oversampling, which is 80 here thus $i=1,2$, $3, \ldots 80$. The PAV of the $i$-th sampling position is defined as $P A V_{i, k}=\max \left(S_{i, k}\right)-\min \left(S_{i, k}\right)$. Then the optimum sampling point of each sub-band signal is obtained which corresponds to the minimum $P A V$, as indicated by the red solid dots in the right column plots of Fig. 3. The complexity of the approach almost linearly proportional to the number of symbol periods considered since only search algorithm is used to obtain the PAV of each sampling position. Through the paper, only 100 symbol periods of samples were used for the timing recovery algorithm. However, this approach still has problem with

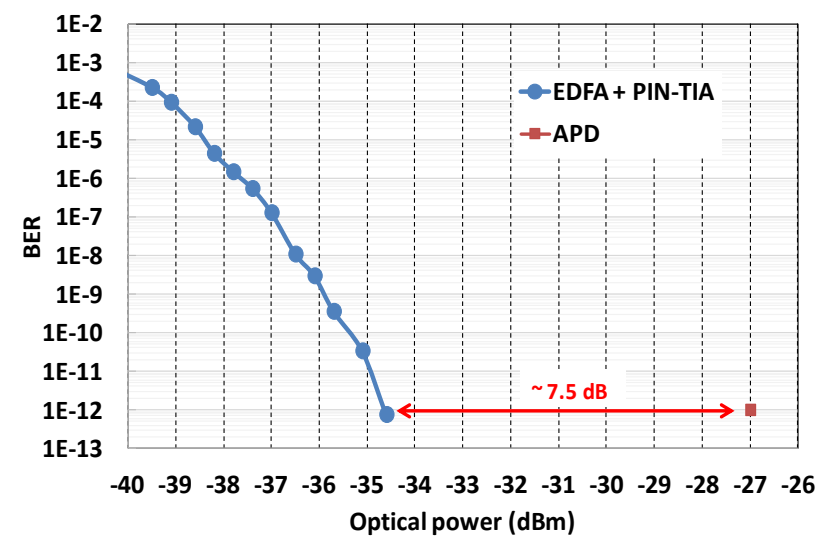

Fig. 5. Receiver optical power sensitivity of a reference $10.3125-\mathrm{Gb} / \mathrm{s} \mathrm{NRZ}$ signal using the $10-\mathrm{G}$ transceiver.

dealing with CAP signals at high frequency sub-bands. As indicated in Fig. 3, the amplitude peak varation property is weakened in the $7^{\text {th }}$ sub-band and beyond. And timing error would easily cause cycle slip issue for the following MMA equalizer.

To efficiently address the cycle slip issue, (partial) differential QAM encoding and decoding schemes can be used due to their natural immunity to phase offset. DBPSK and DQPSK are differential coding schemes widely used in various communication systems. Higher order QAMs can be encoded and decoded in a partial differential manner [29]. Fig. 4 illustrates the encoding process for QAM-8, QAM-16, and QAM-32 used in the experiment. For schemes beyond QAM-32 the principle is identical. The basic idea is to use the first two bits of each symbol to present the phase index $M_{k}$ of each quadrant relative to the first quadrant, namely $M_{k} \in[0,1$, $2,3]$. By differentially encoding

$$
M_{d, t}=\left(M_{d, t-1}+M_{k, t}\right) \bmod 4
$$

where $t$ is the discrete time index, the information bits are contained in the phase difference between two consecutive symbols rather than in the absolute phase, as indicated by arrows in QAM-8 encoder in Fig. 4 (a). Note that for QAM-16 and QAM-32 the two bits are encoded in a similar way so we did not mark it using arrows in Fig. 4(b) and (c). The reminder bits of each symbol are used to map the first quadrant constellation points denoted as $A_{t}$. As shown in Fig. 4, $A_{t} \in$ $[1+\mathrm{j}, 1+\sqrt{ } 2+(1+\sqrt{ } 2) \mathrm{j}], A_{t} \in[1+\mathrm{j}, 3+\mathrm{j}, 1+3 \mathrm{j}, 3+3 \mathrm{j}]$, and $A_{t} \in$ $[3+\mathrm{j}, 5+\mathrm{j}, 1+3 \mathrm{j}, 3+3 \mathrm{j}, 5+3 \mathrm{j}, 1+5 \mathrm{j}, 3+5 \mathrm{j}, 5+5 \mathrm{j}]$ for QAM-8, QAM-16, and QAM-32, respectively. The final encoded QAM symbol at time $t$ is given by $A_{t} \cdot e^{j \frac{\pi}{2} M_{d, t}}$. Due to the differential encoding, the constellation shows deviations from ideal Gray coding for QAM-16 and QAM-32, as indicated by the red circle marks in Fig. 4. This may cause some penalty. In addition, MMA works better with a square QAM-8 or QAM-32 compared with its regular variant [30].

The decoding process is the inverse of the encoder. After blind MMA equalization, a recovered square constellation of QAM- $R(R=8,16$, or 32$)$ for each sub-band is obtained. To obtain the first two bits of the symbol at time $t$, differential 

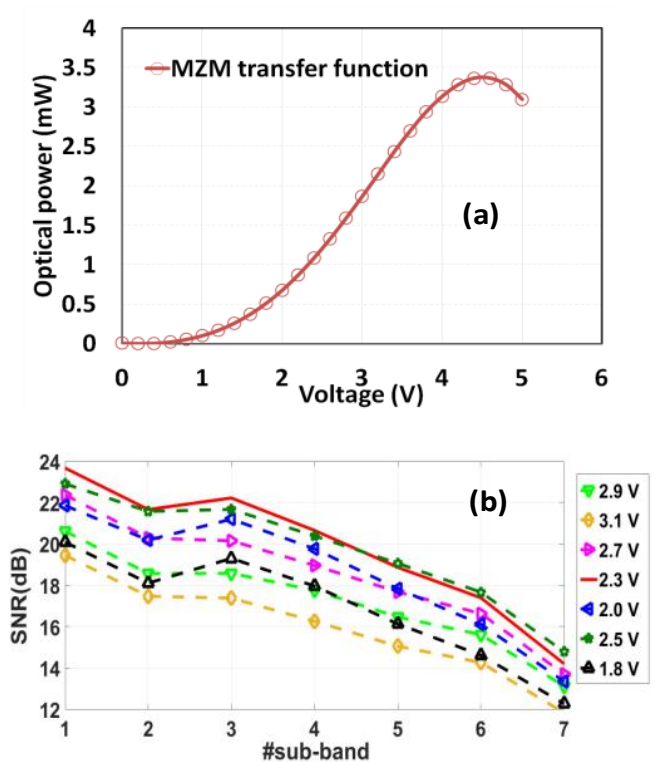

Fig. 6. (a) the measured transfer function of the 10-G TTA, and (b) multi-band CAP sub-band SNR under various driving voltages.

decoding is needed based on

$$
\tilde{M}_{k, t}=\left(\tilde{M}_{d, t}-\tilde{M}_{d, t-1}\right) \bmod 4
$$

The remainder $\left(\log _{2} R-2\right)$ bits of each symbol are recovered according to its corresponding amplitude $A_{t}$ in the first quadrant. The drawback of (partial) differential QAM is that it will cause penalty due to error propagation and non ideal Grey coding. For square QAM- $R$, the theoretical penalty defined as the bit error probability ratio of the differentially coded system to the non-differentially coded system is given by $1+\frac{\log _{2} R}{2(\sqrt{R}-1)}$ [29], which corresponds to $3 \mathrm{~dB}$ and $2.2 \mathrm{~dB}$ for QAM-4 and QAM-16, respectively.

The partial differential QAM encoding/decoding schemes together with MMA equalizer relax significantly the requirement on timing recovery and bring about zero-overhead for receiver DSPs.

Note that, strictly speaking, the above-mentioned timing issue is a timing offset issue. Timing jitter in this context could be defined as the short-time variation of the optimum sampling point. The differential coding itself can only solve the cycle slip problem but will not track phase noise. The blind MMA can track phase noise to some extent if the phase noise causes a constellation phase rotation within $[-\pi / 2, \pi / 2]$, beyond which cycle slip occurs and coding deals with it. In spite of this, it may still cause penalty when jitter occurs since jitter leads to CAP constellations with not only phase rotation (can be corrected) but also with points more scattered [22]. On this point, K-mean or other nonlinear machine learning equalization schemes may offer better performance than linear MMA [31-34]. Note that equalizers alone can not address cycle slip issue. On the other hand, since the system uses direct detection and transversal filters for sub-band signal de-multiplexing, no optical or RF local oscillator is involved. It would not expect a very strong/fast phase noise for such a system.
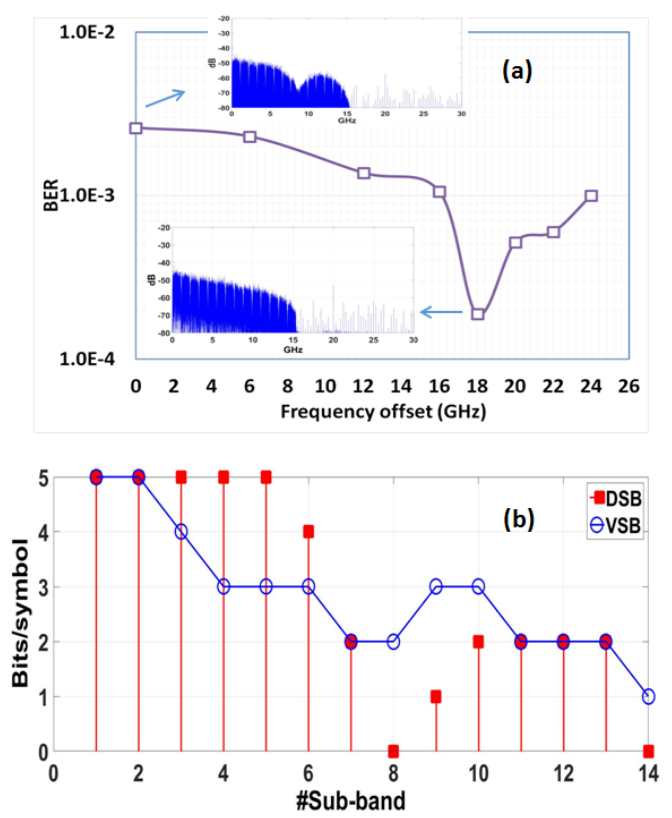

Fig. 7. (a)BER versus optical filtering frequency offset for transmission over 40-km SSMF and (b) the bit loading maps for frequency offset of $0 \mathrm{GHz}$ and $18 \mathrm{GHz}$, respectively.

\section{EXPERIMENTAL RESULTS}

\section{A. 10-G Optical Transceiver}

The 10-G TTA and the pre-amplified PIN-TIA are the key components that enable low cost realization of the $40-\mathrm{Gb} / \mathrm{s}$ LR-PON system proposed here. On one hand, the optical transmitter takes the majority cost of the overall transceiver cost and the cost is approximately proportionally depends on the bandwidth [13]. On the other hand, the low-cost optical transceiver is one of the major limiting factors determining the achievable performance. As shown in Fig. 1 inset, the overall bandwidth is only about 7-GHz for optical back to back case. By using the 10-G transceiver, Fig. 5 presents the optical power sensitivity for a $10.3125-\mathrm{Gb} / \mathrm{s}$ reference NRZ signal. The measurement is based on an optical back-to-back (B2B) setup including a PRBS pattern generator (PPG) at $10.3125 \mathrm{~Gb} / \mathrm{s}$ in the transmitter and a BERT in the receiver. The receiver sensitivity for a BER of $10^{-12}$ for the $10.125-\mathrm{Gb} / \mathrm{s}$ NRZ signal is approximately $-34.5 \mathrm{dBm}$ using no FECs. As a reference, the optical power sensitivity of a commercially available $10-\mathrm{G}$ avalanche photodiode (APD) receiver is also presented in Fig. 5 [35], which shows about 7.5-dB penalty at BER of $10^{-12}$ relative to the EDFA + PIN-TIA receiver. This penalty agrees well with the theoretical estimation made in [11].

Fig. 6(a) presents the measured static response of the TTA used in the experiment, which are obtained by sweeping the bias voltage added to one arm of the MZM via keeping the voltage of the other arm fixed. Fig. 6(b) is the result by applying the RF multi-band CAP signal consisting of seven sub-bands. By observing the sub-band SNR, the optimum bias voltage of $2.3 \mathrm{~V}$ is obtained, which corresponding to the highest SNR that most sub-bands can achieve for an optical B2B link. The optimum bias voltage is almost at the quadrature point which minimizes modulation nonlinearity. It should be mentioned that 


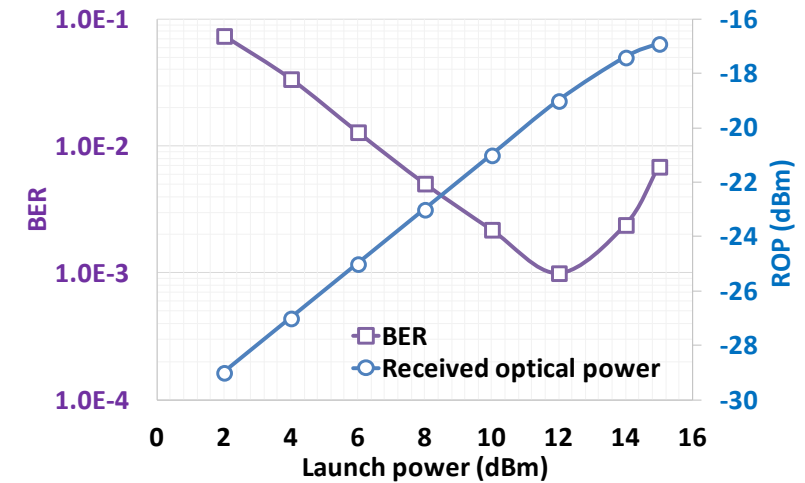

Fig. 8. The dependence of system BER on optical launch power for a 40-km SMF link. The received optical power corresponding to each launch power is also presented.

the MZM supports differential input driving signal, in which case the MZM has zero chirp. However, due to the lack of an RF amplifier with differential output in lab, the MZM is driven by a single-ended multi-band CAP signal. As a result, the MZM behaves negative chirp.

\section{B. Optimization of Optical Filtering}

The OBPFs used in the setup in Fig. 1 can be used to generate a VSB multi-band CAP signal, which is realized by tuning the wavelength of the laser integrated within the TTA. In practice, (De-)MUXs can be used to replace OBPFs. Fig. 7(a) examines the dependence of the system BER performance on the frequency offset between the laser wavelength and the OBPF central wavelength. An optimum frequency offset of 18 $\mathrm{GHz}$ is obtained, below which the BER degradation is due to fiber chromatic dispersion, which causes a frequency notch within the signal spectrum as indicated by the upper inset of Fig. 7(a). Consequently the surrounding sub-bands adjacent to the notch must use low order modulations or even completely dropped off, as shown in the corresponding bit loading map in Fig. 7(b). In order to achieve a fixed bit rate of $40 \mathrm{~Gb} / \mathrm{s}$, low frequency sub-bands have to adopt high order modulation formats such as QAM-32, leading to degradation in optical power sensitivity. The lower inset in Fig. 7(a) shows that the optimum frequency offset enables a signal spectrum without distortions and moderate order modulation schemes are can be allocated to most sub-bands [see Fig. 7(b)], which improves the overall optical power sensitivity. Beyond the optimum frequency offset, performance degradation is attributed to unwanted filtering of the other sideband.

\section{Optimization of Optical Launch Power}

The optical power launched into the fiber link has a straightforward influence on the achievable system power budget. Fig. 8 explores the relationship between system BER performance and optical launch power. It shows that the optimum launch power is approximately $12 \mathrm{dBm}$. Below the optimum launch power the system is limited by achievable received optical power, as indicated in Fig. 8. Further increase in launch power causes Brillouin scattering thus serious system degradation occurs.

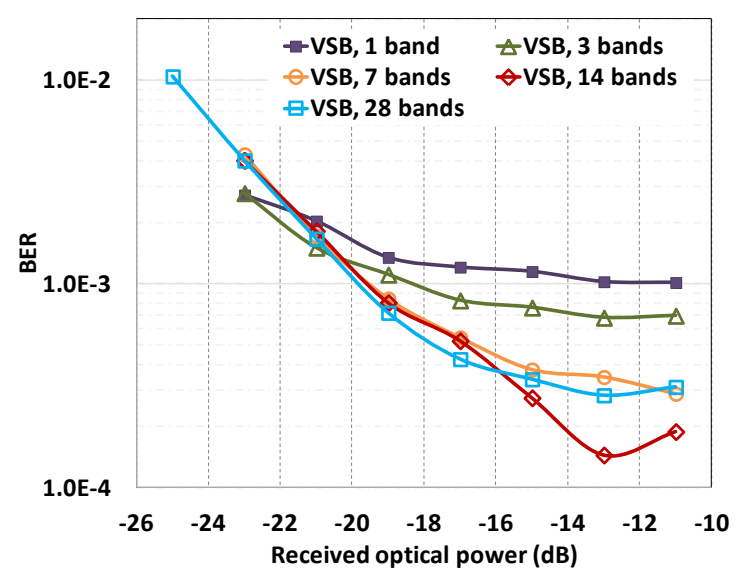

Fig. 9. System BER versus optical power for multi-band CAP having various sub-band count. Results are based on 40-km SMF link and asymmetric optical filtering with $18 \mathrm{GHz}$ frequency offset.

\section{Effect of Sub-band Count}

The sub-band count of multi-CAP is of important concern regarding system performance and DSP complexity [24]. Fig. 9 looks into the effect of sub-band count on the receiver optical power sensitivity. The performance developing trend indicates that the error floor reduces with increasing sub-band count and a sub-band count of 14 brings about the lowest error floor. The physics underpinning such trend is because more sub-bands enable the system with smaller granularity to better adapt itself to the channel conditions. Meantime, the optical power sensitivity at BER of $10^{-3}$ increases with increasing sub-band count and saturates at sub-band count of 7 . This is attributed to two reasons: the use of an optical VSB configuration and the stronger sub-band $\times$ sub-band intermixing presents for a larger sub-band count. Overall, a sub-band count of 14 brings about both optimum power sensitivity and error floor, which is used for the following measurement.

\section{E. Tolerance to Timing Error}

Based on the optimum parameters obtained above, this sub-section evaluates the system tolerance to timing error ( $\mathrm{T}$ E) by using the coding and algorithms introduced in Section II. Fig. 10 illustrates the BER variations with the TE for

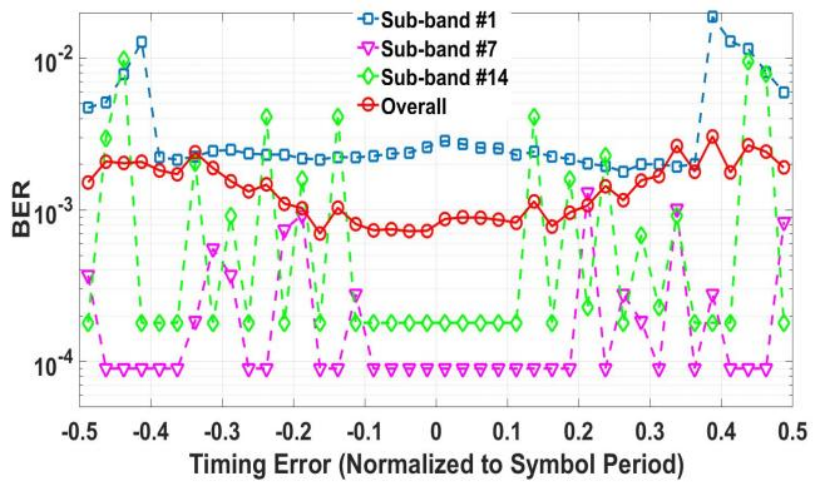

Fig. 10. Sub-band and overall BER versus timing error normaliyed to symbol period. Results are based on 40-km SMF link with asymmetric optical filtering with $18 \mathrm{GHz}$ frequency offset and a received optical power of $-19 \mathrm{dBm}$. Note that, for sub-bands 7 and 14 , the lowest BER is actually 0 which is replaced with a fixed BER value for display convenience. 

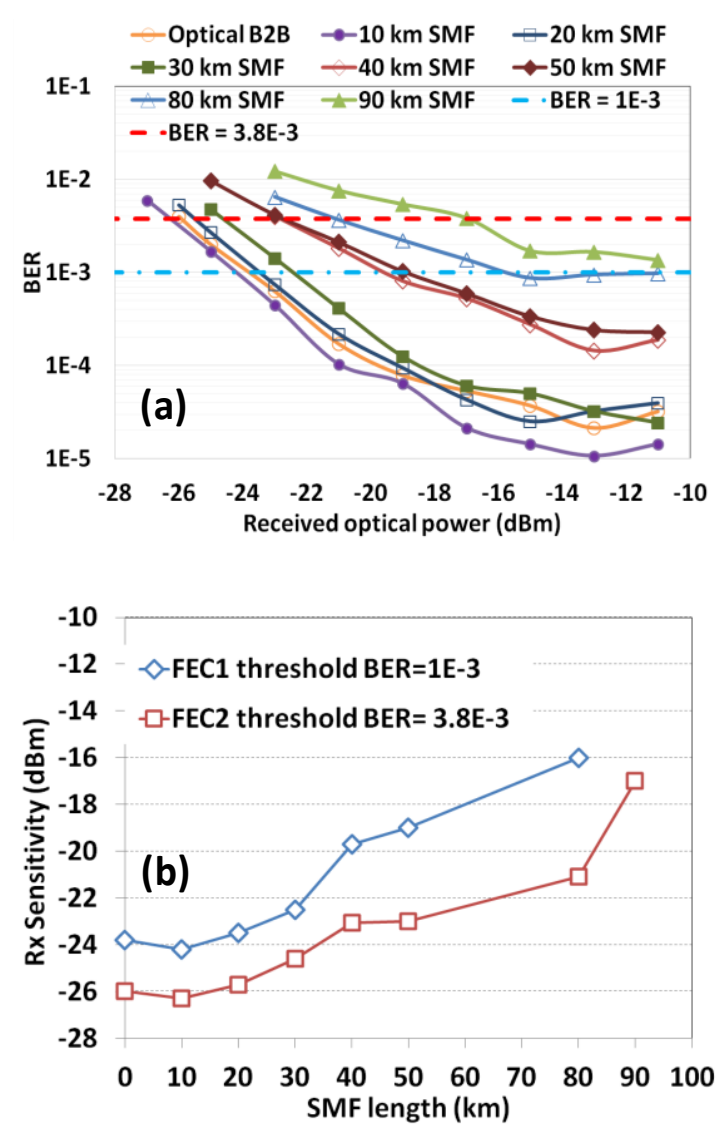

Fig. 11. (a) BER versus received optical power at various fiber lengths and (b) receiver optical power sensitivity versus fiber length considering the two FEC schemes.

representative sub-band signals and the overall signal. The TE here is defined as the timing offset relative to the optimum sampling point obtained via the timing recovery, which is artificially generated in order to obtain Fig. 10. As the blind MMA equalizer has a tap space of half symbol period, the BERs show nearly symmetrical variation over timing error. As shown in Fig. 10, sub-band BERs (overall BER) show limited BER variation below $2 \times 10^{-2}$ (below $3 \times 10^{-3}$ ) within a TE range of -0.5 to 0.5 symbol periods. This verifies that the proposed differential coding schemes successfully addressed the cycle slip issue in an efficient manner by comparing Fig. 2 and Fig. 10. It is very interesting to see that there is a TE range around zero TE where sub-band BERs and overall BER keep the lowest, which can be named absolute tolerant TE range. Excellent absolute tolerant TE rang of at least \pm 0.1 symbol period is obtained even for the highest frequency CAP sub-band. Generally speaking, the absolute tolerant TE range is larger for sub-band at lower frequency, as indicated in Fig. 10. This is not suprising by considering the eye quality shown in Fig. 3.

In addition, the absolute tolerant TE range for sub-band 1 almost centered at zero $\mathrm{TE}$, which means the timing recovery successfully extracted the optimum sampling point based on the signal amplitude statistical property. However, the absolute tolerant TE range for sub-band 7 shows significant center offset relative to zero $\mathrm{TE}$, indicating that the timing recovery algorithm may fail to obtain the optimum sampling point due to
TABLE I

OPTICAL LINK POWER BUDGETS AT DIFFERENT FIBER LENGTHS

\begin{tabular}{|c|c|c|}
\hline FIBER LENGTH $(\mathrm{km})$ & 80 & 90 \\
\hline LAUNCH POWER $(\mathrm{d} B \mathrm{~m})$ & 12 & 12 \\
\hline LINK POWER BUDGET $(d B)$ [FEC1] & 28 & - \\
\hline LINK POWER BUDGET $(d B)$ [FEC2] & 33.1 & 29 \\
\hline
\end{tabular}

the weakened amplitude statistical property [see Fig. 3(b)]. As a result, cycle slip occurs by comparing Fig. 2 and Fig. 10. Thanks to the differential coding, the requirement on timing recovery is largely relaxed. It must be pointed that, equalizers alone could not address cycle slip issue, neither can offer such excellent absolute tolerant TE ranges [33].

\section{F. BER Performance}

The experimental measurements presented here are obtained by the optimized link parameters obtained in sub-section III-A to III-D. Based on the optimum conditions, Fig. 11(a) shows the BER versus received optical power performance for VSB multi-band CAP signal at different fiber lengths. Successful transmission of $40 \mathrm{~Gb} / \mathrm{s}$ multi-CAP signals over 80-km SMF is achieved by considering both FEC schemes and $90-\mathrm{km}$ SMF transmission is feasible only when FEC with threshold BER of $3.8 \times 10^{-3}$ is used. The receiver sensitivity at different fiber lengths is shown in Fig. 11(b) by considering the two FEC schemes. The general trend is that the longer the transmission distance, the larger penalty it has, except for $10-\mathrm{km}$ SMF case where the system exhibits negative penalty. Beyond $10 \mathrm{~km}$ the system has positive penalty which increases with increasing fiber length. The appearance of negative penalty at around 10-km SMF is attributed to the negative chirp of the InP MZM configured with a single-ended driving input [11], which can offset part of the fiber chromatic dispersion. On the other hand, the error floor also increases with increasing fiber length.

\section{G. Estimation of Power Budgets}

Table I summarizes the achievable optical link power budgets for an optical amplified LR 40-Gb/s NG-PON using multi-band CAP for transmission distances up to $90 \mathrm{~km}$. The power budget is defined as the power ratio between the optical launch power and the received optical power prior to pre-amplifier EDFA required at the FEC threshold BER. By using the FEC with BER threshold of $10^{-3}$, an $80-\mathrm{km}$ SMF transmission is feasible with a power budget of $28 \mathrm{~dB}$ but it fails to support longer transmission distances. By considering a stronger and also more complex FEC with BER threshold of $3.8 \times 10^{-3}$, transmissions over both $80-\mathrm{km}$ and $90-\mathrm{km}$ SMFs are successful with an optical power budget of $33.1 \mathrm{~dB}$ and $29 \mathrm{~dB}$, respectively. It should be noted that, for short fiber lengths less than $40 \mathrm{~km}$, in order to save cost, the downlink pre-amplifier EDFA can be removed and the PIN receiver can be replaced by an APD and the uplink transmitters can use TTAs without booster EDFAs. When using an APD, the receiver shows about $7.5 \mathrm{~dB}$ less sensitivity compared with an EDFA + PIN receiver, as indicated in Fig. 5 and [11]. A TTA without booster EDFA can only offer approximately $3 \mathrm{dBm}$ launch power. Therefore, the power budget of lower cost short fiber link $(<40 \mathrm{~km})$ is slightly smaller. 


\section{CONCLUSIONS}

In conclusion, an optical amplified $40 \mathrm{~Gb} / \mathrm{s}$ lane rate LR-PON using multi-band CAP has been demonstrated by using 10-G class transceiver only. Detailed analysis of the timing jitter issue for multi-band CAP is presented. A simple timing recovery approach, partial differential QAM encoding and decoding schemes, together with blind MMA equalization were proposed to not only address the timing issue efficiently but also facilitate zero-overhead receiver DSPs. By optimizing the critical system parameters, results show that $80-\mathrm{km}(90-\mathrm{km})$ SMF transmission is feasible with a link power budget of $33 \mathrm{~dB}$ ( $29 \mathrm{~dB})$ by considering a FEC threshold BER of $3.8 \times 10^{-3}$.

\section{ACKNOWLEDGMENT}

This work was partly supported by the European Union (EU) under a Marie Curie Intra-European Fellowship for Career Development via the project CEEOALAN (grant number 623515) and EU's Horizon 2020 research and innovation programme under the Marie Curie project EPIC (grant number 713567). The authors would also like to thank Dr. Helmut Griesser, Dr. Annika Dochhan, and Dr. Nicklas Eiselt from ADVA Optical Networking SE, Germany, for useful discussions and suggestions.

\section{REFERENCES}

[1] Recommendation ITU-T G.989 series, 2013.

[2] IEEE 802.3 Ethernet Working Group, "Industry connections feasibility assessment for the next generation of EPON," online (2015).

[3] T. Asai, "5G radio access network and its requirements on mobile optical network," Proc. ONDM, Pisa, 2015.

[4] T. Pfeiffer, and F. Schaich: "Optical architectures for mobile back- and fronthauling," OFC Wireless backhauling workshop, Los Angeles (2012).

[5] Z. Li, L. Yi, X. Wang, and W. Hu, "28 Gb/s duobinary signal transmission over $40 \mathrm{~km}$ based on $10 \mathrm{GHz}$ DML and PIN for $100 \mathrm{~Gb} / \mathrm{s}$ PON," Opt. Express. Vol. 23, no. 16, p. 20249-20256, Aug. 2015.

[6] J. Yu, Z. Jia, M.-F. Huang, M. Haris, P.N. Ji, T. Wang, and G.-K. Chang, "Applications of 40-Gb/s chirp-managed laser in access and metro networks,” J. Lightw. Technol., vol. 27, no. 3, pp. 253-265, Feb. 2009.

[7] H. Ji, L. Yi, Y. Li, L. Xue, X. Li, Q. Yang, S. Wang, Y. Yang, S. Yu, and W. Hu, "Field Demonstration of a Real-Time 100-Gb/s PON Based on 10G-Class Optical Devices,” J. Lightwav. Technol., vol. 35, no. 10, pp. 1914-1921, May 2017.

[8] V. Houtsma, and D. V. Veen, "Demonstration of symmetrical $25 \mathrm{Gbps}$ TDM-PON with $31.5 \mathrm{~dB}$ optical power budget using only $10 \mathrm{Gbps}$ optical components," ECOC, Paper PDP. 4.3, Sept. 2015.

[9] V. Houtsma, D. V. Veen, A. Gnauck, and P. Iannone, "APD-based Duobinary direct detection receivers for 40 Gbps TDM-PON," Proc. OFC, Paper Th4H. 1, Los Angeles, US, Mar. 2015.

[10] Z. Ye, S. Li, N. Cheng, and X. Liu, "Demonstration of high-performance cost-effective 100-Gb/s TWDM-PON using 4x25-Gb/s optical Duobinary channels with $16-\mathrm{GHz}$ APD and receiver-side post-equalization" ECOC, Paper Mo.3.4.4, Sept. 2015

[11] J. L. Wei, N. Eiselt, H. Griesser, K. Grobe, M. Eiselt, J. J. Vegas-Olmos, I. T. Monroy, J.-P. Elbers, "Demonstration of the First Real-Time End-to-End 40-Gb/s PAM-4 for Next-Generation Access Applications using 10-Gb/s Transmitter," J. Lightw. Techol., vol. 34, no. 7, pp. 1628-1635, Apr. 2016

[12] M. Ruffini, “Metro-Access Network Convergence," Proc. of OFC, Paper Th4B.1, Mar. 2016.

[13] J. L. Wei, K. Grobe, C. Sánches, E. Giacoumidis, and H. Griesser,, "Comparison of cost- and energy-efficient signal modulations for next generation passive optical networks," Opt. Express, vol. 23, no. 22, pp. 28271-28281, Nov. 2015.
[14] J. L. Wei, K. Grobe, C. Wagner, E. Giacoumidis, and H. Griesser, "40 Gb/s Lane Rate NG-PON using Electrical/Optical Duobinary, PAM-4 and Low Complex Equalizations," Proc. of OFC, Paper Tu3C.5, 2016.

[15] D.-Z. Hsu, C.-C. Wei, H.-Y. Chen, W.-Y. Li, and J. Chen, "Cost-effective 33-Gbps intensity modulation direct detection multi-band OFDM LR-PON system employing a 10-GHz-based transceiver," Opt. Express, vol. 19, no. 18, pp. 17546-17556, Aug. 2011.

[16] D. Qian, N. Cvijetic, J. Hu, and T. Wang, "108 Gb/s OFDMA-PON With Polarization Multiplexing and Direct Detection,” J. Lightw. Technol., vol. 28, no. 4, pp 484-493, Feb. 2010.

[17] J. Wei, N. Eiselt, C. Sanchez, R. Du, and H. Griesser, "56 Gb/s multi-band CAP for data center interconnects up to an $80 \mathrm{~km} \mathrm{SMF,"} \mathrm{Opt.}$ Lett., vol. 42, no. 17, pp. 4122-4125, 2016.

[18] J. Zhang, J. Yu, F. Li, N. Chi, Z. Dong, and X. Li, " $11 \times 5 \times 9.3 \mathrm{~Gb} / \mathrm{s}$ WDM-CAP-PON based on optical single-side band multi-level multi-band carrier-less amplitude and phase modulation with direct detection," Opt. Express, vol. 21, no. 16, pp. 18842-18848, Aug. 2013.

[19] J. Wei, "DSP-Based Multi-Band Schemes for High Speed Next Generation Optical Access Networks," Proc. OFC, Paper M3H.3, Los Angeles, US, Mar. 2017.

[20] C. C. Wei, K.-Z. Che, L.-W. Chen, C.-Y. Lin, W.-J. Huang, and J. Chen "High-Capacity Carrierless Amplitude and Phase Modulation for WDM Long-Reach PON Featuring High Loss Budget, ” J. Lightwav. Technol., vol. 35, no. 4, pp. 1075-1082, Feb. 2017.

[21] Z. Dong, J. Yu, and J. Lu, "Bandwidth-Efficient WDM-CAP-PON Using Digital Hilbert Single-Sideband Modulation,” IEEE Photon. J., vol. 7, no. 5, pp. 7903907, Oct. 2015.

[22] J. Wei, Q. Cheng, D. G. Cunningham, R. V. Penty, and I. H. White, "100 Gb/s Hybrid Multiband (HMB) CAP/QAM signal transmission over a single wavelength," J. Lightwav. Technol., vol. 33, no. 2, pp. 415-423, Jan. 2015.

[23] N. Eiselt, J. Wei, H. Griesser, A. Dochhan, M. H. Eiselt, J.-P. Elbers, J. J. V. Olmos, and I. T. Monroy, "Evaluation of Real-Time 8x56.25 Gb/s (400G) PAM-4 for Inter-Data Center Application over $80 \mathrm{~km}$ of SSMF at 1550 nm,” J. Lightwav. Technol., vol. 35, no. 5, pp. 955-962, Feb. 2017.

[24] J. L. Wei, C. Sanchez, and E. Giacoumidis, "Fair Comparison of Complexity between Multi-band CAP and DMT for Data Center Interconnects," Opt. Lett., vol. 42, no. 19, pp. 3860-3863, Oct. 2017.

[25] ITU-T Recommendation G.975.1. 2004.

[26] L. M. Garth, J. Yang, and J. -J. Werner, "Blind equalization algorithms for dual-mode CAP-QAM reception," IEEE Trans. Commun., vol. 49, no. 3, pp. 455-466, Mar. 2001.

[27] J.-T. Yuan, and K.-D. Tsai, "Analysis of the Multimodulus Blind Equalization Algorithm in QAM Communication Systems, " IEEE Trans. Commun., vol. 53, no. 9, pp. 1427-1431, Sept. 2005.

[28] M. Oerder, and H. Meyr, "Digital filter and square timing recovery", IEEE Trans. On. Comm., vol. 36, no. 5, pp. 605-612, May 1988.

[29] W. J. Weber, "Differential Encoding for Multiple Amplitude and Phase Shift Keying Systems," IEEE Trans. Commun. Vol. 26, no. 3, pp. 385-391, Mar. 1978.

[30] X. Zhou, L. E. Nelson, P. Magill, B. Zhu, and D. W. Peckham, "8x450-Gb/s,50-GHz-Spaced,PDM-32QAM transmission over 400km and one 50GHz-grid ROADM," Proc. OFC, paper PDPB, 2011.

[31] A. Tatarczak, M. I. Olmedo, T. Zuo, J. Estaran, J. B. Jensen, X. Xu, and I. T. Monroy, "Enabling 4-lane Based 400 G Client-Side Transmission Links with MultiCAP Modulation," Advances in Opt. Technol., Vol. 2015, Paper ID 935309, 2015.

[32] R. Puerta, J. J. Vegas Olmos, I. T. Monroy, N. N. Ledentsov, and J. P. Turkiewicz, "Flexible MultiCAP Modulation and its Application to 850 nm VCSEL-MMF Links, ” J. Lightwav. Technol., vol. 35, no. 15, pp. 3168-3173, Aug. 2017.

[33] M. I. Olmedo, T. Zuo, J. B. Jensen, Q. Zhong, X. Xu, S. Popov, and I. T. Monroy "Multiband carrierless amplitude phase modulation for high capacity optical data links," Journal of Lightwave Technology, vol. 32, no. 4, pp. 798-804, 2014.

[34] E. Giacoumidis, A. Matin, J. L. Wei, N. J. Doran, and X. Wang, "Blind Nonlinearity Equalization by Machine Learning based Clustering for Single- and Multi-Channel Coherent Optical OFDM," accepted by J. Lightwav. Technol. for publication, Nov. 2017.

[35] An example commercial 10-G APD. Available at https://www.neophotonics.com/product/10g-apd/ 
Jinlong Wei (S'09-M'11-SM'17) is currently a senior researcher in Huawei Technologies Duesseldorf GmbH, European Research Center, Germany. He received his $\mathrm{PhD}$ degree in Electronic Engineering from Bangor University, Bangor, UK in 2010 and worked there afterwards as a post-doc researcher before joining the Centre for Photonics Systems of Cambridge University, UK as a research associate in 2011. He was awarded a Marie Curie fellowship and joined ADVA Optical Networking SE, Germany as a senior engineer in Sept. 2014. He has participated in about 10 European national and international projects as well as projects with industry and realized a number of world-first system demonstrations with his colleagues. He has been contributing to the next generation 100 Gigabit Ethernet study within IEEE802.3. He has authored and coauthored over 120 journal and conference publications and 5 public patents.

Elias Giacoumidis information is not available at the time of submission. 